\title{
Analysis of microbiota in first episode psychosis identifies preliminary associations with symptom severity and treatment response
}

\section{Schwarz, Emanuel}

2018-02

Schwarz , E , Maukonen , J , Hyytiäinen , T , Kieseppä , T , Oresic , M , Sabunciyan , S , Mantere , O , Saarela , M , Yolken , R \& Suvisaari , J 2018, ' Analysis of microbiota in first episode psychosis identifies preliminary associations with symptom severity and treatment response ' , Schizophrenia Research , vol. 192 , pp. 398-403 . https://doi.org/10.1016/j.schres.2017.04.017

http://hdl.handle.net/10138/301092

https://doi.org/10.1016/j.schres.2017.04.017

publishedVersion

Downloaded from Helda, University of Helsinki institutional repository.

This is an electronic reprint of the original article.

This reprint may differ from the original in pagination and typographic detail.

Please cite the original version. 


\title{
Analysis of microbiota in first episode psychosis identifies preliminary associations with symptom severity and treatment response
}

\author{
Emanuel Schwarz ${ }^{\mathrm{a}, *, 1}$, Johanna Maukonen ${ }^{\mathrm{b}, 1}$, Tiina Hyytiäinen ${ }^{\mathrm{b}}$, Tuula Kieseppä ${ }^{\mathrm{c}}$, Matej Orešič ${ }^{\mathrm{f}, 2}$, \\ Sarven Sabunciyan ${ }^{\mathrm{d}}$, Outi Mantere ${ }^{\mathrm{g}, 3}$, Maria Saarela $^{\mathrm{b}}$, Robert Yolken ${ }^{\mathrm{d}}$, Jaana Suvisaari ${ }^{\mathrm{e}}$ \\ a Department of Psychiatry and Psychotherapy, Central Institute of Mental Health, Medical Faculty Mannheim, Heidelberg University, Mannheim, Germany \\ b VTT Technical Research Centre of Finland Ltd., Espoo, Finland \\ c University of Helsinki and Helsinki University Hospital, Psychiatry, Helsinki, Finland \\ d Stanley Neurovirology Laboratory, Johns Hopkins School of Medicine, Baltimore, MD, USA \\ e National Institute for Health and Welfare (THL), Helsinki, Finland \\ ${ }^{\mathrm{f}}$ Turku Centre for Biotechnology, University of Turku and Åbo Akademi University, FI-20520 Turku, Finland \\ g Bipolar Disorders Clinic, Douglas Mental Health University Institute, Montréal, Canada
}

\section{A R T I C L E I N F O}

\section{Article history:}

Received 19 December 2016

Received in revised form 4 April 2017

Accepted 8 April 2017

Available online 22 April 2017

\section{Keywords:}

Schizophrenia

Microbiome

Psychosis

Response

\begin{abstract}
A B S T R A C T
The effects of gut microbiota on the central nervous system, along its possible role in mental disorders, have received increasing attention. Here we investigated differences in fecal microbiota between 28 patients with firstepisode psychosis (FEP) and 16 healthy matched controls and explored whether such differences were associated with response after up to 12 months of treatment. Numbers of Lactobacillus group bacteria were elevated in FEP-patients and significantly correlated with severity along different symptom domains. A subgroup of FEP patients with the strongest microbiota differences also showed poorer response after up to 12 months of treatment. The present findings support the involvement of microbiota alterations in psychotic illness and may provide the basis for exploring the benefit of their modulation on treatment response and remission.
\end{abstract}

(C) 2017 Elsevier B.V. All rights reserved.

\section{Introduction}

The human commensal microbiota plays an important role for the host physiological, nutritional, and immunological processes (Hooper et al., 2001). It is involved in the production of short-chain fatty acids (SCFA) and gases, the transformation of bile acids, the formation of vitamins, and the potential formation of mutagenic, toxic, and carcinogenic substances (O'Hara and Shanahan, 2006). Commensal bacteria are able to modulate the expression of host genes that regulate diverse and fundamental physiological functions. Furthermore, it is being increasingly recognized that the gut-brain axis provides a bidirectional route of communication that employs neural, hormonal and immunological pathways, and that dysfunction of this axis can have pathophysiological consequences (Collins et al., 2012; Cryan and Dinan, 2012).

Converging evidence suggests that gastrointestinal (GI) inflammation may have an important role in psychotic disorders (Dinan et al.,

\footnotetext{
* Corresponding author.

E-mail address: emanuel.schwarz@zi-mannheim.de (E. Schwarz).

1 These authors contributed equally to this work.

2 Present address: Steno Diabetes Center, Gentofte, Denmark.

3 Present address: Centre de recherche de l'institut universitaire en santé mentale Douglas, Montreal, Canada.
}

2014). Autoimmune diseases associated with chronic gastrointestinal inflammation, like celiac disease, Crohn's disease, and ulcerative colitis, are associated with increased risk of schizophrenia (Benros et al., 2011) and mood disorders (Benros et al., 2013). Antibodies to anti-Saccharomyces cerevisiae, a marker of GI-inflammation commonly observed in celiac disease and in Crohn's disease (Shor et al., 2012; Viitasalo et al., 2014), are elevated in people with first-episode psychosis (FEP), schizophrenia, and bipolar disorder (Severance et al., 2012; Severance, Gressitt, et al. 2014). Furthermore, markers of GI-inflammation correlate with serum levels of C-reactive protein (Severance et al., 2013), suggesting that GI-inflammation may contribute to systemic low-level inflammation, a common feature in psychotic disorders (Bergink et al., 2014). A contributing factor may also be an increased GI-permeability (Severance et al., 2016).

The effects of gut microbiota on the central nervous system, along with its possible role in mental disorders, have received increasing attention in recent years (Borre et al., 2014). Animal studies have shown that gut microbiota influence brain and anxiety- and depression-related behavior, and the vagus nerve seems to be an important mediator in these associations (Cryan and Dinan, 2012; Bravo et al., 2011; Bercik et al., 2011). A link between gut microbiota and autistic-like behaviors has been supported by animal studies (Hsiao et al., 2013; de Theije et al., 2014). Similarly, human studies suggest an association with risk of 
autism and major depressive disorder (Bravo et al., 2011). Here we investigated the composition of fecal microbiota of FEP-patients and compare it to healthy matched controls. We also explore whether the detected compositional differences were associated with symptom severity and remission after 2 months and 1 year of treatment.

\section{Methods}

\subsection{Participants}

Patients with first psychotic episode (FEP, 18-40 years), were recruited from the catchment area of the Helsinki University Hospital, Finland. All primary psychotic disorders were included, substance-induced psychoses and psychotic disorders due to a general medical condition were excluded. The inclusion criterion was a score of at least 4 in the items assessing delusions (Unusual Thought Content) or hallucinations in the Brief Psychiatric Rating Scale - Extended (BPRS-E) (Ventura et al., 1993). Controls, matched by age, sex and region of residence, were identified from the Population Register Center and sent an invitation letter to participate in the study.

All participants provided written informed consent for protocols approved by the Ethics committee of the Hospital District of Helsinki and Uusimaa (257/13/03/03/2009). The treating psychiatrist evaluated the patients' capacity to provide informed consent before they were approached by the study team.

\subsection{Clinical assessment}

FEP-patients were assessed three times. The baseline assessment was conducted when patients entered treatment and was able to give informed consent. Follow-ups were conducted 2 and 12 months thereafter. Fecal samples were analyzed at baseline only. Clinical information was utilized from baseline and follow-up to evaluate whether baseline fecal microbiota composition predicted clinical outcome at follow-ups.

Each assessment point consisted of an interview, questionnaire, and collection of blood and fecal samples. The severity of psychotic symptoms was assessed using the BPRS-E complemented by three domains (alogia, anhedonia-asociality and avolition-apathy) from the Scale for the Assessment of Negative Symptoms (SANS) (Andreasen et al., 2010). For assessing the severity of positive symptoms, we calculated sum of BPRS items Hallucinations, Unusual thought content, Bizarre behavior and Conceptual disorganization; and for assessing the severity of negative symptoms, we calculated sum of BPRS item Blunted affect and SANS items Alogia, Anhedonia and Avolition. BPRS items were rescaled from 1-7 to 0-6 for calculating the sum scores. In addition, the BPRS total score was used to assess the current (past week) severity of symptoms, and the remission criteria by Andreasen et al. (2005) to assess remission at follow-ups (the 6-month duration was not required). Global Assessment of Functioning (GAF) was used to assess current functioning. Structured Clinical Interview for DSM-IV was conducted at 2- and 12-month follow-ups, and for the final diagnostic assessment, also medical records from all psychiatric treatment contacts were reviewed. Study interviews were conducted by trained research nurses or psychologists, and all interviews were reviewed together with a senior psychiatrist (Prof. Jaana Suvisaari) before assigning the DSM-IV diagnosis.

Food habits were assessed by questions adapted from the postal survey "Health Behavior and Health among the Finnish Adult Population" (AVTK) (Männistö et al., 2010). Specific questions are shown in Supplementary Table 1.

Physical activity was assessed using the Gothenburg scale (Dinan et al., 2014). Based on these ratings, we created a dichotomous variable with " 0 " indicating all subjects who did not engage in physical activity during their leisure time and with "1" for physically active subjects.

Patients and controls were assessed for several metabolic parameters. Laboratory methods for their measurement have been described previously (Keinänen et al., 2015).

\subsection{Fecal samples}

Fresh fecal samples from hospital patients were taken at baseline, the next morning after the initial interview and stored immediately at $-80^{\circ} \mathrm{C}$. Outpatients visited the laboratory within one week after the interview and similarly had fresh samples taken in the same morning to the laboratory for immediate storage at $-80^{\circ} \mathrm{C}$ Control people also took the samples at home and delivered them within a few hours to the laboratory for immediate storage at $-80^{\circ} \mathrm{C}$. Fecal samples were defacated into a larger sampling bowl, from which duplicate samples (if possible) were further transferred to smaller tubes for storage. Fecal samples were not collected if the subject reported having any of the following: antibiotic use during the past 3 months, chronic gastrointestinal disease, gastrointestinal surgery, or diagnosed celiac disease. Fecal samples were not collected during follow-up visits.

\subsection{Quantitative real-time PCR ( $q P C R$ ) analyses}

DNA extraction was performed from $0.2 \mathrm{~g}$ of fecal sample as previously described (Maukonen et al., 2006). The fecal bacterial numbers were analyzed by performing seven different bacterial qPCR analyses for "all" bacteria, Lachnospiraceae (Eubacterium rectale group; Erec), Ruminococcaceae (Clostridium leptum group; Clept), Bacteroides spp., Atopobium group in addition to bifidobacteria and Lactobacillus-group (comprising of the genera Lactobacillus, Leuconostoc, Pediococcus, and Weissella) as previously described (Maukonen et al., 2006; Simões et al., 2013). Briefly, standard curves were obtained from genomic DNA templates isolated from pure cultures as previously described (Simões et al., 2013). The extracted DNA was quantified by using NanoDrop 2000c equipment (Thermo Scientific). For each bacterium of interest, the number of cells present in the volume loaded to the qPCR reaction was calculated on the basis of the genome size and the respective $16 \mathrm{~S}$ ribosomal RNA copy number per cell, identified through the National Center for Biotechnology Information genome database (http://www. ncbi.nlm.nih.gov/genome/).

\subsection{Metagenomic analyses}

Aliquots of 75-100 ng of extracted fecal DNA were used to generate paired end libraries using the Nugen Ultralow DR Multiplex System following the manufacturer's instructions (http://info.nugeninc.com/ UGOvationUltralowDRMultiplexSystem1-96.html). The libraries were purified and run on the Bioanalyzer to confirm size and concentration. Libraries were then sequenced using the Illumina Hi Seq 2000 generating approximately $200,000,000$ paired end reads of 100 nucleotides in length. At least 100,000,000 reads were obtained from each individual.

Sequence reads were filtered to remove low quality sequences, resulting in a minimum length of 60 nucleotides. Those with homology to human sequences were removed in 2 stages. The first stage employed the program Bowtie (http://bowtie-bio.sourceforge.net/index.shtml). A sliding window approach was used to align a $40 \mathrm{bp}$ subsequence from the reads to the human genome Build 37 (ftp://ftp.ccb.jhu.edu/pub/ data/bowtie_indexes/hg18.ebwt.zip). During each iteration, reads mapping to the human genome were removed and the subsequence used for alignment was offset by 5 bases. The reads that survived this subtraction procedure were then imported into CLC Genomics Workbench Version 6 (www.clcbio.com) and a second subtraction against the human genome build 37 and additional sets of human sequences available from the UCSC Genome Browser (http://genome.ucsc.edu) was performed using the Reference Mapping algorithm employing global alignment with the settings of similarity $=0.4$ and length $=0.4$. In some cases duplicate reads were also removed by the use of CLC. The sequence reads which remained following the removal of the human sequences were then aligned to the Refseq Bacterial Database (ftp://ftp.ncbi.nlm.nih.gov/refseq/release/bacteria/), version available 
on 10-30-2012, using the Read Mapping algorithm of the CLC Genomics Workbench Version 6 and settings of length fraction $=0.8$ and similarity $=0.8$. Consensus reads generated from these mappings were retained for further analysis.

\subsection{Statistical analysis}

To perform metagenomics analysis, we used the linear discriminant analysis (LDA) effect size (LEfSe) method (http://huttenhower.sph. harvard.edu/lefse/) for biomarker discovery, which performs a combined assessment of statistical significance and biological relevance. The method utilizes non-parametric Kruskal-Wallis test to investigate case-control differences, using a sample-wise normalized matrix of relative abundances and determines the effect size of a given taxon using LDA. We performed this analysis using the default settings (alpha $=$ 0.05 , effect-size threshold of 2 ). We used as abundance measure the length of the consensus sequence created by the sample and the reference bacterial genome.

To identify potential patient subgroup effects based on metagenomic microbial abundance, we use the most significant bacterial families from the LEfSe method and perform partial least squares regression analysis on taxon-wise normalized data to the range of abundances (Milligan and Cooper, 1988). Using the projection on the first two components, we identified the patients that showed the strongest difference in microbiota profiles compared to controls. The patient clusters were subsequently assessed for differences in clinical scores and frequency of remission after 2 and 12 months of treatment. This was performed using logistic regression with age and sex as covariates. All statistical analyses for metagenomic data were performed using the software package R (http://cran.r-project.org/).

Differences in sociodemographic and clinical characteristics were assessed with Wilcoxon Rank sum tests or Fisher's exact tests. Student's $t$-test was used for testing group differences in $\log _{10}$ transformed qPCR derived variables and associations with patients' symptoms were quantified using Spearman's correlation tests.

\section{Results}

\subsection{Clinical characteristics and qPCR-analyses}

The cohort investigated in this study comprised 28 FEP-patients and 16 controls (Table 1$)$, which did not differ in terms of age $(P=0.25)$, sex $(P=0.76)$ and several metabolic parameters (Table 1$)$. Half of the patients received a DSM-IV diagnosis of schizophrenia by one-year follow-up, while four had schizophreniform disorder, one schizoaffective disorder, two bipolar I disorder with psychotic features, one major depressive disorder with psychotic features and six psychotic disorder not otherwise specified. Olanzapine $(n=10)$, risperidone $(n=7)$ and quetiapine $(n=8)$ were the most commonly used antipsychotics. The median duration of antipsychotic treatment at baseline was 20 days (first quartile 13, third quartile 46). Two patients who had refused antipsychotic treatment were antipsychotic-naïve at the baseline assessment.

The numbers of bacteria within the analyzed bacterial groups were similar in both patients and controls (Fig. 1). Due to high inter-individual variation and small study population, the differences between cases and controls were not statistically significant (Fig. 1). However, bacterial numbers correlated with symptom severity in patients (Table 2). Such correlations were seen for bacterial numbers in the Lactobacillus group, Lachnospiraceae, Ruminococcaceae and Bacteroides spp. and for the predominant bacterial number, and the strongest correlations were seen for higher level of negative symptoms and poorer functioning. Notably, duration of antipsychotic treatment did not correlate significantly with bacterial numbers in any of the studied groups.
Table 1

Demographic and metabolic characteristics of the investigated cohort (BL: baseline, $2 \mathrm{~m}$ : 2 month follow-up, $12 \mathrm{~m}$ : 12 months follow-up). Values are shown as mean $\pm S D$.

\begin{tabular}{|c|c|c|c|}
\hline & $\begin{array}{l}\text { FEP patients } \\
(n=28)\end{array}$ & $\begin{array}{l}\text { Controls } \\
(n=16)\end{array}$ & $\begin{array}{l}P \text {-value }\left(\chi^{2} \text { or }\right. \\
\text { Mann-Whitney } U)\end{array}$ \\
\hline Men/women & $16 / 12$ & $8 / 8$ & 0.65 \\
\hline Age & $25.9 \pm 5.5$ & $27.8 \pm 6.0$ & 0.25 \\
\hline BMI & $23.8 \pm 4.3$ & $23.9 \pm 3.1$ & 0.50 \\
\hline Schizophrenia (yes/no) & $14 / 14$ & - & - \\
\hline GAF & $35.1 \pm 4.7$ & - & - \\
\hline $\begin{array}{l}\text { BPRS positive } \\
\text { symptoms }\end{array}$ & $6.8 \pm 3.5$ & - & - \\
\hline $\begin{array}{l}\text { BPRS negative } \\
\text { symptoms at BL }\end{array}$ & $6.0 \pm 3.8$ & - & - \\
\hline $\begin{array}{l}\text { BPRS positive } \\
\text { symptoms at } 2 \mathrm{~m}\end{array}$ & $3.6 \pm 4.7$ & - & - \\
\hline $\begin{array}{l}\text { BPRS negative } \\
\text { symptoms at } 2 \mathrm{~m}\end{array}$ & $6.3 \pm 4.1$ & - & - \\
\hline $\begin{array}{l}\text { BPRS positive } \\
\text { symptoms at } 12 \mathrm{~m}\end{array}$ & $1.6 \pm 2.9$ & - & - \\
\hline $\begin{array}{l}\text { BPRS negative } \\
\text { symptoms at } 12 \mathrm{~m}\end{array}$ & $4.5 \pm 4.4$ & - & - \\
\hline S-hs-CRP mg/l & $1.9 \pm 2.3$ & $2.3 \pm 3.2$ & 0.75 \\
\hline P-glucose mmol/l & $4.8 \pm 1.7$ & $4.0 \pm 0.5$ & 0.15 \\
\hline S-insulin mU/l & $20.1 \pm 27.9$ & $8.3 \pm 4.6$ & 0.22 \\
\hline S-cholesterol mmol/l & $4.7 \pm 0.8$ & $4.8 \pm 1.1$ & 0.71 \\
\hline S-HDL-C mmol/l & $1.4 \pm 0.3$ & $1.6 \pm 0.5$ & 0.48 \\
\hline S-LDL-C mmol/l & $2.8 \pm 0.8$ & $2.7 \pm 0.8$ & 0.66 \\
\hline S-triglycerides mmol/l & $1.2 \pm 0.8$ & $1.2 \pm 1.2$ & 0.84 \\
\hline
\end{tabular}

BMI, body mass index; hs-CRP, high sensitivity C-reactive protein; HDL-C, high density lipoprotein cholesterol; LDL-C, low density lipoprotein cholesterol; $\mathrm{P}=$ plasma, $\mathrm{S}=$ serum.

\subsection{Metagenomic analysis}

Application of the LefSe method identified 5 significant differences at the family level and 10 significant differences at the genus level. At the family level, Lactobacillaceae, Halothiobacillaceae, Brucellaceae and Micrococcineae were increased whereas Veillonellaceae were decreased in FEP patients compared to controls. Among genera, we found statistically significant FEP increases in Lactobacillus, Tropheryma, Halothiobacillus, Saccharophagus, Ochrobactrum, Deferribacter and Halorubrum. In contrast, Anabaena, Nitrosospira and Gallionella showed decreased levels. Fig. 1a-c indicate that Lactobacillaceae were overrepresented among the taxa that were most strongly increased in patients.

We found schizophrenia patients to be significantly less physically active compared to control subjects ( $P=0.003$, Fisher's exact test). Since this can impact on microbiota composition, we repeated the LefSe analysis using only physically active subjects ( 15 cases, 16 controls). In this smaller subject group, we also identified significant patient increases in Lactobacillaceae and significant decreases in Veillonellaceae (Supplementary Fig. 1).

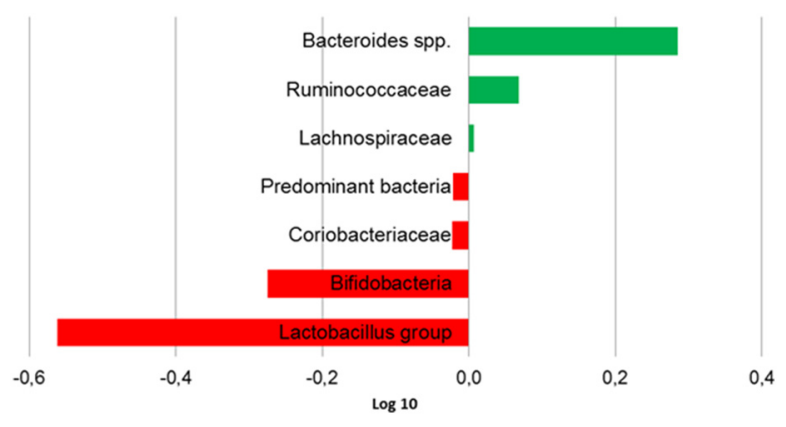

Fig. 1. Difference in bacterial numbers $(\log 10)$ as detected with bacterial group specific qPCR analyses between healthy controls and FEP patients. Bacterial groups that shower enriched levels in patients are shown in red, whereas those with enriched numbers in controls are shown in green. 
Table 2

Spearman rank order correlations between bacterial numbers and clinical symptoms in first-episode psychosis patients.

\begin{tabular}{|c|c|c|c|c|c|}
\hline Group & $\begin{array}{l}\text { BPRS total score } \\
\text { rho ( } P \text { value })\end{array}$ & $\begin{array}{l}\text { Positive symptoms } \\
\text { rho ( } P \text { value })\end{array}$ & $\begin{array}{l}\text { Negative symptoms } \\
\text { rho ( } P \text { value })\end{array}$ & $\begin{array}{l}\mathrm{GAF} \\
\text { rho ( } P \text { value })\end{array}$ & $\begin{array}{l}\text { Duration of antipsychotic treatment } \\
\text { rho }(P \text { value })\end{array}$ \\
\hline Lachnospiraceae & $0.38(0.048)$ & $0.15(0.45)$ & $0.49(0.008)$ & $-0.17(0.38)$ & $0.07(0.73)$ \\
\hline Ruminococcaceae & $0.32(0.10)$ & $0.17(0.38)$ & $0.47(0.011)$ & $-0.39(0.040)$ & $-0.03(0.89)$ \\
\hline Bacteroides spp. & $0.38(0.049)$ & $0.34(0.07)$ & $0.23(0.25)$ & $-0.40(0.034)$ & $-0.17(0.38)$ \\
\hline Coriobacteriaceae & $0.27(0.17)$ & $0.26(0.18)$ & $0.06(0.76)$ & $-0.13(0.51)$ & $0.02(0.91)$ \\
\hline Bifidobacteria & $0.30(0.13)$ & $0.22(0.26)$ & $0.36(0.06)$ & $-0.23(0.25)$ & $0.21(0.29)$ \\
\hline Lactobacillus group & $0.48(0.009)$ & $0.47(0.012)$ & $0.37(0.05)$ & $-0.52(0.004)$ & $0.02(0.94)$ \\
\hline Predominant bacteria & $0.35(0.07)$ & $0.21(0.28)$ & $0.42(0.027)$ & $-0.42(0.027)$ & $-0.13(0.50)$ \\
\hline
\end{tabular}

\subsubsection{Associations with symptom severity, clinical response and potential} confounders

To investigate associations with clinical symptom severity and remission, we identified patients with the greatest multivariate difference in microbiota composition compared to controls. For this, we performed partial least squares regression using the five bacterial families that were identified as showing a significant case-control difference by the LefSe method. We specifically compared the 18 patients that clustered outside the distribution of the controls against the remaining patients
(Fig. 2a). Logistic regression accounting for the effects of age and gender identified significant associations with negative symptoms and GAF scores $(P=0.0327$ and $P=0.0495$, respectively, Fig. $2 \mathrm{~b}$ and $\mathrm{c})$, but not with positive symptoms $(P=0.331)$. (See Fig. 3 .)

At 12 months follow-up, data for 19 out of 28 patients was available and we carried the last observed response forward (including follow-up assessment at 2 months). We found that microbiota clustering at intake was significantly associated with remission at follow-up $(P=0.014$, logistic regression). Among patients that clustered with controls in a)

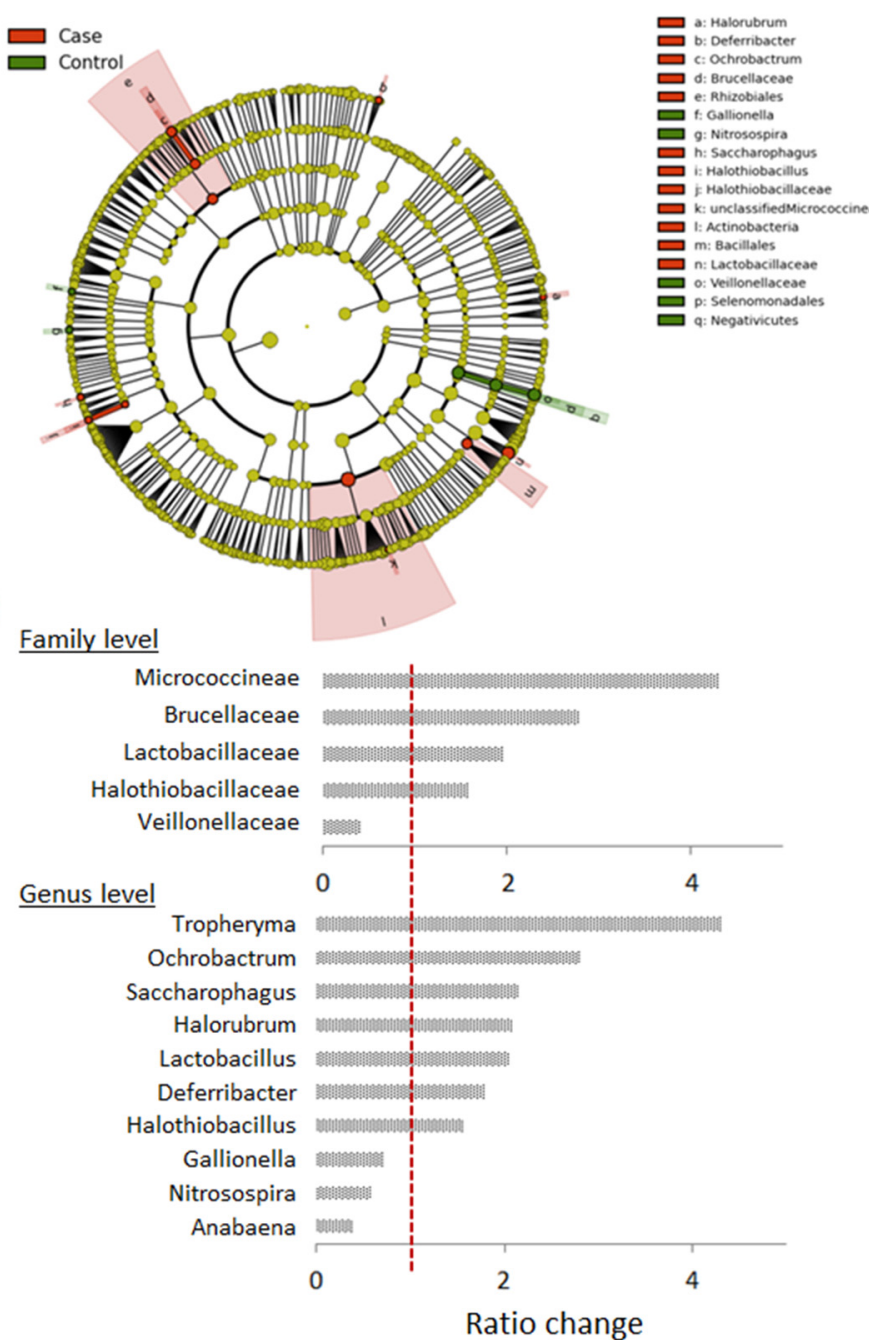

b)

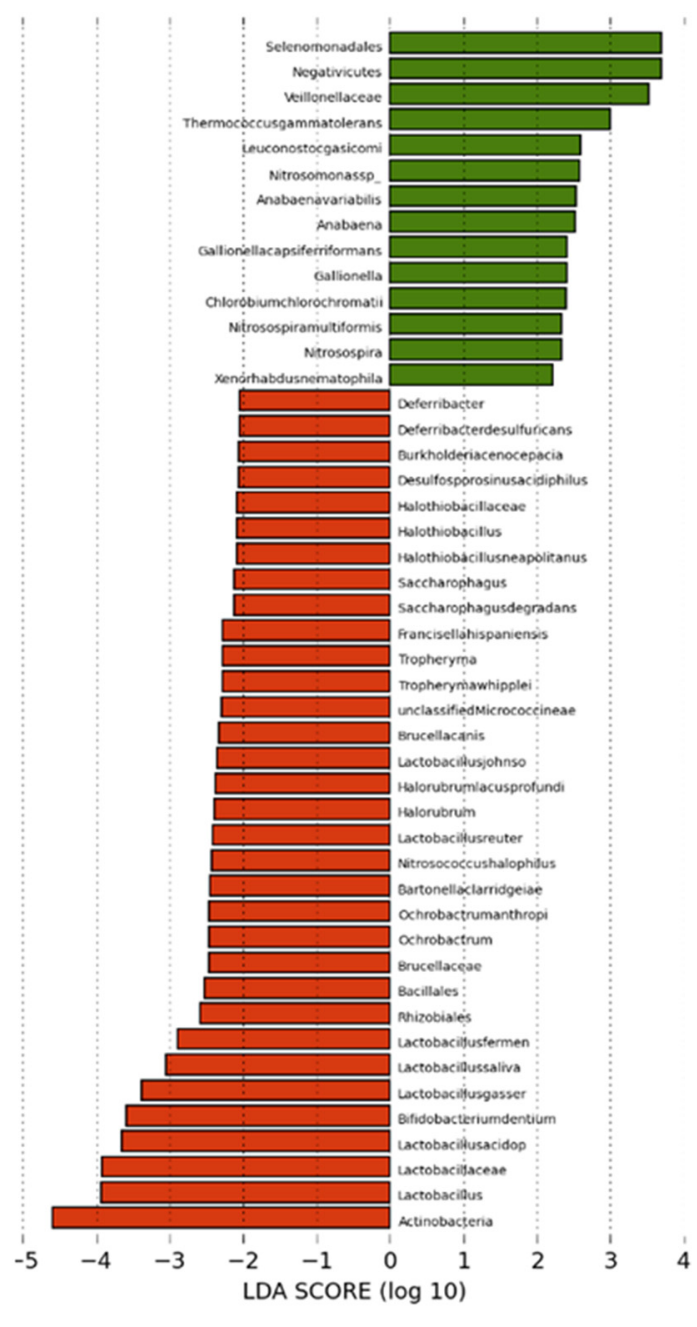

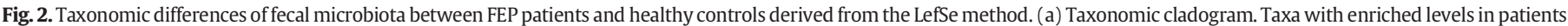

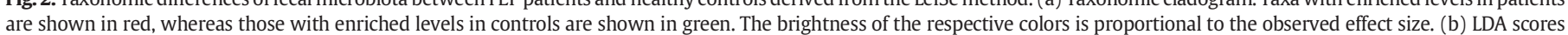
observed for individual taxa that passed the LefSe significance threshold. (c) Ratio changes (patient levels/control levels) at the family and genus level. 


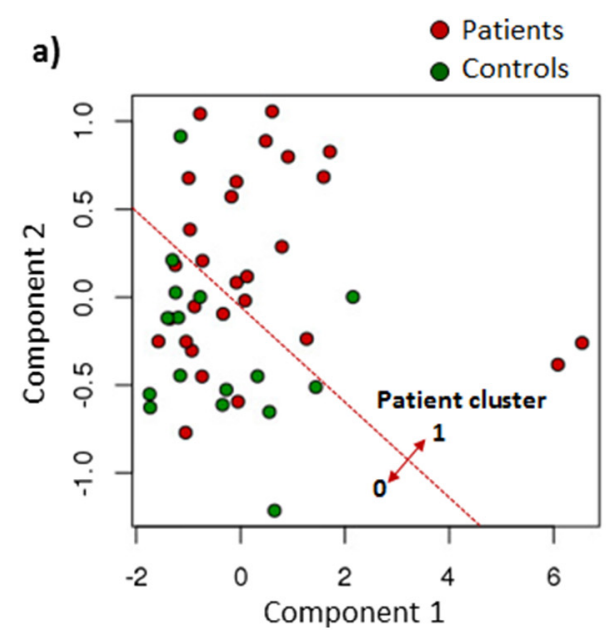

b)

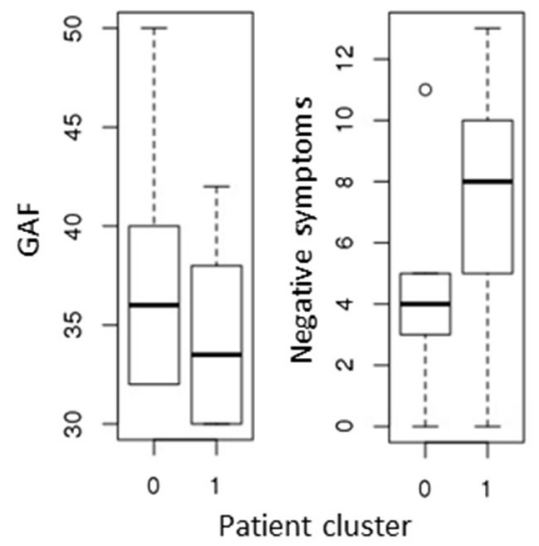

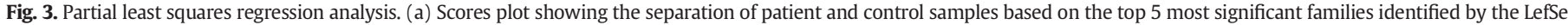
method. (b) Boxplots showing differences in GAF and negative symptom BPRS scores at baseline between patients in clusters 1 and 2 (identified from score plot in a).

terms of microbiota composition, $70 \%$ showed remission after 12 months, whereas only $28 \%$ of patients with "abnormal" microbiota composition experienced remission. To explore whether symptom severity at baseline was a potential confounder for the associations between microbiota clustering and remission, for example due to the fact that more severely ill patients that had a differential microbiota profile received more intensive treatment, we included baseline GAF scores as covariate. However, this did not lead to a substantial decrease in the association strength between clustering and remission $(P=0.021$, logistic regression). Investigating the 2 month follow-up time-point individually showed a trend towards association between microbiota clustering and remission ( $P=0.09$, logistic regression).

To exclude confounding, we compared the clustering against other variables of potential interest and found no associations with physical activity $(P=0.38)$, BMI $(P=0.47)$, type of psychosis (schizophrenia vs other, $P=0.99)$, duration of antipsychotic treatment $(P=0.46)$ or the distribution of risperidone, quetiapine or olanzapine treatment ( $P=0.99, P=0.40$ and $P=0.70$, respectively). Finally, we compared the microbiota clustering in patients against 15 ratings quantifying the intake of different food types over the week prior to sample collection (Supplementary Table 1). This did not identify any significant associations with the clustering (minimum $P$-value across 14 comparisons was 0.07).

\section{Discussion}

The present analysis identified differences between FEP-patients and controls in the gut microbial composition and Lactobacillaceae emerged as one of the families with the strongest alterations. Lactobacillus group bacterial numbers correlated positively with severity of psychotic symptoms and negatively with global assessment of functioning. The increased levels of lactobacilli in FEP patients are consistent with reports in autistic children (Adams et al., 2011) and with reports showing increased abundance of bacteriophage genome of Lactobacillus phage phiadh in human oropharyngeal samples of schizophrenia patients (Yolken et al., 2015).

Besides Lactobacillus group bacteria, Lachnospiraceae, Ruminococcaceae, Bacteroides spp. and predominant bacteria correlated negatively with global assessment of functioning. Interestingly, Blautia spp., Roseburia spp. and Lachnospiraceae incertae sedis, all belonging to Lachnospiraceae, have been shown to be decreased in major depressive disorder (Jiang et al., 2015). On the other hand, Bacteroides spp., Faecalibacterium sp. and Ruminococcus spp. have been shown to be less abundant in major depressive disorder (Jiang et al., 2015) and decreased fractional representation of Faecalibacterium has also been found in bipolar disorder (Evans et al., 2017). Likewise we detected a trend that FEP patients our control group had lower numbers of Bacteroides spp. and Ruminococcaceae (including Faecalibacterium sp. and Ruminococcus spp.).

At the family level, relative proportions of Lactobacillaceae, Halothiobacillaceae, Brucellaceae and Micrococcineae were increased whereas Veillonellaceae were decreased in our FEP patients compared to controls. Lower relative proportion of Veillonellaceae has also been detected in major depressive disorder (Jiang et al., 2015).

We found worse current global functioning as well as increased negative symptom BPRS scores in a patient cluster that showed an abnormal microbiota profile. In addition, within this patient cluster, remission during follow-up was substantially less frequent compared to patients with a microbiota profile similar to controls. We did not find evidence that this effect was due to differences in diet or treatment of more severely ill patients As the findings are similar to those reported in MDD and autism, gut microbiota might reflect some dietary pattern, treatment, or etiological factors shared by several psychiatric disorders, or other associated characteristics such as obesity. On the other hand, it may be speculated that a normalization of the microbiota composition is a potential protective factor for schizophrenia. Interestingly, in mice, ingestion of Lactobacillus rhamnosus decreases anxiety and depressionlike behavior and reduces the stress-induced increase of plasma corticosterone levels (Bravo et al., 2011). However, it should be kept in mind that probiotic lactobacilli by definition are "live microorganisms which when administered in adequate amounts confer a health benefit on the host" (Araya et al., 2002), and they do not usually colonize the GItract, as compared to commensal lactobacilli. Nevertheless, probiotic usage should be considered as a potential confounder for future investigations.

The main limitation of the present study is its small sample size. Therefore, findings should be seen as preliminary and need to be reproduced in independent samples. As the present microbiota profile was substantially driven by Lactobacillaceae levels, which have previously been shown to correlate with the consumed diet (Cuervo et al., 2014; Zhang et al., 2013; Hooda et al., 2012), diet may impact on the microbiota composition and, in turn, clinical symptom severity and remission. Here, we did not identify significant associations of dietary habits with patient clustering, more detailed dietary data would have been needed to conclusively exclude bias. Also, it is known from rodent studies that antipsychotic treatment can impact on microbiota profiles (Davey et al., 2012). Although the median duration of antipsychotic treatment was only 20 days, all patients were treated, and therefore, potential bias cannot be excluded in the present study. Furthermore, we observed a significant difference in physical activity between patients 
and controls. In a smaller subsample stratified for physical activity, Lactobacillaceae and Veillonellaceae were still associated with diagnosis, but many of the less significant strains from the full cohort could not be found. While this may partially be influenced by sample size difference, future studies should pay special attention to careful matching regarding physical activity.

In conclusion, we identified significant yet preliminary differences in microbial numbers and composition between FEP patients and controls that showed associations with symptom severity at baseline and response after 12 months of treatment. Further studies are required to replicate these findings and may provide the basis for exploring the benefit of microbiota modulation on treatment response and remission.

Supplementary data to this article can be found online at http://dx. doi.org/10.1016/j.schres.2017.04.017.

\section{Author contributions}

JM, TK, MO, OM, MS, RY, JS, and ES conceived and designed the experiments. JM, TH, SS, TK, OM and JS performed the experiments. ES, JM, and JS analyzed the data. ES, JM, MS, RY, and JS contributed reagents/materials/analysis tools. All authors contributed to the writing of the manuscript.

\section{Conflict of interests}

The authors declare no conflicts of interest.

\section{Role of the funding source}

The sponsor had no role in study design; in the collection, analysis and interpretation of data; in the writing of the report; or in the decision to submit the paper for publication.

\section{Acknowledgements}

This study was supported by Jalmari and Rauha Ahokas Foundation (TH), Stanley Medical Research Institute (RY and SS, JM), Sigrid Juselius Foundation (JS), Finnish Cultural Foundation (JS), Helsinki University Central Hospital (OM), Academy of Finland (Grant \# 278171 to JS), and the Deutsche Forschungsgemeinschaft (DFG) Emmy-Noether-Program SCHW 1768/1-1 (ES). The funders had no role in study design, data collection and analysis, decision to publish, or preparation of the manuscript. Marja-Liisa Jalovaara and Tuula Mononen are greatly acknowledged for their excellent technical assistance.

\section{References}

Hooper, L.V., et al., 2001. Molecular analysis of commensal host-microbial relations hips in the intestine. Science 291 (5505), 881-884.

Adams, J.B., et al., 2011. Gastrointestinal flora and gastrointestinal status in children with autism-comparisons to typical children and correlation with autism severity. BMC Gastroenterol. 11, 22.

Andreasen, N.C., et al., 2005. Remission in schizophrenia: proposed criteria and rationale for consensus. Am. J. Psychiatr. 162 (3), 441-449.

Andreasen, N.C., et al., 2010. Antipsychotic dose equivalents and dose-years: a standardized method for comparing exposure to different drugs. Biol. Psychiatry 67 (3) 255-262.

Araya, M., 2002. Joint FAO/WHO Working Group - Guidelines for the Evaluation of Probiotics in Food

Benros, M.E., et al., 2011. Autoimmune diseases and severe infections as risk factors for schizophrenia: a 30-year population-based register study. Am. J. Psychiatr. 168 (12), 1303-1310.

Benros, M.E., et al., 2013. Autoimmune diseases and severe infections as risk factors for mood disorders: a nationwide study. JAMA Psychiatry 70 (8), 812-820.

Bercik, P., et al., 2011. The anxiolytic effect of Bifidobacterium longum NCC3001 involves vagal pathways for gut-brain communication. Neurogastroenterol. Motil. 23 (12) $1132-1139$
Bergink, V., Gibney, S.M., Drexhage, H.A., 2014. Autoimmunity, inflammation, and psychosis: a search for peripheral markers. Biol. Psychiatry 75 (4), 324-331.

Borre, Y.E., et al., 2014. Microbiota and neurodevelopmental windows: implications for brain disorders. Trends Mol. Med. 20 (9), 509-518.

Bravo, J.A., et al., 2011. Ingestion of Lactobacillus strain regulates emotional behavior and central GABA receptor expression in a mouse via the vagus nerve. Proc. Natl. Acad. Sci. U. S. A. 108 (38), 16050-16055.

Collins, S.M., Surette, M., Bercik, P., 2012. The interplay between the intestinal microbiota and the brain. Nat. Rev. Microbiol. 10 (11), 735-742.

Cryan, J.F., Dinan, T.G., 2012. Mind-altering microorganisms: the impact of the gut microbiota on brain and behaviour. Nat. Rev. Neurosci. 13 (10), 701-712.

Cuervo, A., et al., 2014. Pilot study of diet and microbiota: interactive associations of fibers and polyphenols with human intestinal bacteria. J. Agric. Food Chem. 62 (23), 5330-5336.

Davey, K.J., et al., 2012. Gender-dependent consequences of chronic olanzapine in the rat: effects on body weight, inflammatory, metabolic and microbiota parameters. Psychopharmacology 221 (1), 155-169.

Dinan, T., Borre, Y., Cryan, J., 2014. Genomics of schizophrenia: time to consider the gut microbiome? Mol. Psychiatry 19 (12), 1252-1257.

Evans, S.J., et al., 2017. The gut microbiome composition associates with bipolar disorder and illness severity. J. Psychiatr. Res. 87, 23-29.

Hooda, S., et al., 2012. 454 pyrosequencing reveals a shift in fecal microbiota of healthy adult men consuming polydextrose or soluble corn fiber. J. Nutr. 142 (7), 1259-1265.

Hsiao, E.Y., et al., 2013. Microbiota modulate behavioral and physiological abnormalities associated with neurodevelopmental disorders. Cell 155 (7), 1451-1463.

Jiang, H., et al., 2015. Altered fecal microbiota composition in patients with major depressive disorder. Brain Behav. Immun. 48, 186-194.

Keinänen, J., et al., 2015. Early insulin resistance predicts weight gain and waist circumference increase in first-episode psychosis - a one year follow-up study. Schizophr. Res. 169 (1-3), 458-463.

Männistö, S., et al., 2010. Monitoring diet and diet-related chronic disease risk factors in Finland. Public Health Nutr. 13 (6A), 907-914.

Maukonen, J., et al., 2006. PCR DGGE and RT-PCR DGGE show diversity and short-term temporal stability in the Clostridium coccoides-Eubacterium rectale group in the human intestinal microbiota. FEMS Microbiol. Ecol. 58 (3), 517-528.

Milligan, G.W., Cooper, M.C., 1988. A study of standardization of variables in cluster analysis. J. Classif. 5 (2), 181-204.

O'Hara, A.M., Shanahan, F., 2006. The gut flora as a forgotten organ. EMBO Rep. 7 (7), 688-693.

Severance, E.G., et al., 2012. Gastrointestinal inflammation and associated immune activation in schizophrenia. Schizophr. Res. 138 (1), 48-53.

Severance, E.G., et al., 2013. Discordant patterns of bacterial translocation markers and implications for innate immune imbalances in schizophrenia. Schizophr. Res. 148 (1-3), 130-137.

Severance, E.G., Gressitt, K.L., et al., 2014. Seroreactive marker for inflammatory bowel disease and associations with antibodies to dietary proteins in bipolar disorder. Bipolar Disord 16 (3), 230-240.

Severance, E.G., Yolken, R.H., Eaton, W.W., 2016. Autoimmune diseases, gastrointestinal disorders and the microbiome in schizophrenia: more than a gut feeling. Schizophr. Res. 176 (1), 23-35.

Shor, D.B.-A., et al., 2012. Gastrointestinal-associated autoantibodies in different autoimmune diseases. Am. J. Clin. Exper. Immunol. 1 (1), 49-55.

Simões, C.D., et al., 2013. Habitual dietary intake is associated with stool microbiota composition in monozygotic twins. J. Nutr. 143 (4), 417-423.

de Theije, C.G.M., et al., 2014. Altered gut microbiota and activity in a murine model of autism spectrum disorders. Brain Behav. Immun. 37, 197-206.

Ventura, J., et al., 1993. Brief psychiatric rating scale (BPRS), expanded version (4.0): scales, anchor points, and administration manual. Int. J. Methods Psychiatr. Res. 3, 227-243.

Viitasalo, L., et al., 2014. Early microbial markers of celiac disease. J. Clin. Gastroenterol. 48 (7), 620-624.

Yolken, R.H., et al., 2015. Metagenomic sequencing indicates that the oropharyngeal phageome of individuals with schizophrenia differs from that of controls. Schizophr. Bull. 41 (5), 1153-1161.

Zhang, C., et al., 2013. Structural modulation of gut microbiota in life-long calorie-restricted mice. Nat. Commun. 4, 2163. 